International Journal of Pure and Applied Mathematics

Volume 83 No. $4 \quad$ 2013, 527-537

ISSN: 1311-8080 (printed version); ISSN: 1314-3395 (on-line version)

url: http://www.ijpam.eu

doi: http://dx.doi.org/10.12732/ijpam.v83i4.1

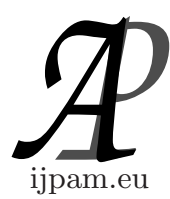

\title{
LOW SEPARATION AXIOMS IN MINIMAL STRUCTURES
}

\author{
Jesús Ávila ${ }^{1 \S}$, Erika Durán ${ }^{2}$, Ximena Moya ${ }^{3}$ \\ ${ }^{1,2,3}$ Department of Mathematics and Statistics \\ University of Tolima \\ Ibagué, COLOMBIA
}

\begin{abstract}
In this work we generalize some separation notions between $T_{0}$ and $T_{1}$ to $m$-spaces. In addition, we study the inclusion relations between the corresponding spaces and prove that the resultant diagram is different from the one in the topological case. Finally, we use the concepts of $m$-kernel, $m$-shell, $m$-derived and $m$-closure to characterize the new separation notions.
\end{abstract}

AMS Subject Classification: 54A05, 54D10

Key Words: separation axioms, $m$-spaces, $m$-open, $m$-closed, $m$-kernel, $m$-shell, $m$-derived, $m$-closure

\section{Introduction}

Separation axioms constitute a classical topic in General Topology. Its systematic study began with the works of Urysohn in 1925 ([8]). Van Est and Freudenthal ([9]) studied in more detail some axioms stronger than $T_{1}$.

The development of the separation axioms between $T_{0}$ and $T_{1}$ started with the work of Young in 1943 ([11]). Later in 1961, Aull and Thron ([1]) introduced new axioms and found that they all can be described in terms of the derived set of singletons.

Received: August 4, 2012

(c) 2013 Academic Publications, Ltd. url: www.acadpubl.eu

${ }^{\S}$ Correspondence author 
The study of more general concepts than the topological structure has taken several directions over the past fifteen years. Maki in 1996 ([4]), studied minimal structures (or $m$-structures) on a set $X$, that is, collections of subsets of $X$ containing the empty set and $X$, with no other restriction. On the other hand, Császár since 1997 has studied topological notions in collections which are closed under unions ([3]). They constitute the well-known generalized topologies.

Many classical topological notions have been studied in $m$-spaces and generalized topologies (see [5], [6], [2], [7], [10] and the literature quoted therein). However, the study of separation axioms have been limited to $T_{0}, T_{1}$ and stronger conditions. This paper is, nevertheless, devoted to the study of some low separation axioms between $m-T_{0}$ and $m-T_{1}$.

In Section 1 we present the most important separation axioms between $T_{0}$ and $T_{1}$ studied in [1] and some basic concepts of $m$-spaces. Following [1], in Section 2 we define some separation notions between $m-T_{0}$ and $m-T_{1}$ and study the inclusion relations between the corresponding spaces. Finally, in Section 3, we use the concepts of $m$-kernel, $m$-shell, $m$-derived and $m$-closure to characterize the new separation notions.

\section{Preliminaries}

In this work we will use the classical notations for the derived and the closure of a set. We start with the well known axioms between $T_{0}$ and $T_{1}$ defined in [1]. We shall simply $x$ to denote the singleton $\{x\}$. In addition, we use the notation $A \vdash B$ to indicate that there exists an open set $G$ such that $A \subseteq G$ and $G \cap B=\emptyset$. Moreover, the derived set and the closure set of $A$ will be denoted by $\operatorname{Der}(A)$ and $C l(A)$ respectively.

Definition 1. The topological space $(X, \tau)$ is called:

1. $T_{0}$ if for each $p, q \in X(p \neq q)$, there exists an open set $G$ such that $p \in G$, $q \notin G$ or $q \in G, p \notin G$.

2. $T_{1}$ if for each $p, q \in X(p \neq q)$, there exist open sets $G, H$ such that $p \in G, q \notin G$ and $q \in H, p \notin H$.

3. $T_{D}$ if for each $x \in X, \operatorname{Der}(x)$ is closed.

4. $T_{U D}$ if for each $x \in X, \operatorname{Der}(x)$ is the union of disjoint closed sets.

5. $T_{D D}$ if it is $T_{D}$ and for each $x, y \in X(x \neq y), \operatorname{Der}(x) \cap \operatorname{Der}(y)=\emptyset$. 
6. $T_{F}$ if for any point $\mathrm{x}$ and any finite subset $F$ of $X$ such that $x \notin F$, either $x \vdash F$ or $F \vdash x$.

7. $T_{F F}$ if for any two finite subsets $F_{1}$ and $F_{2}$ of $X$ with $F_{1} \cap F_{2}=\emptyset$, either $F_{1} \vdash F_{2}$ or $F_{2} \vdash F_{1}$.

8. $T_{Y}$ if for each $x, y \in X(x \neq y), C l(x) \cap C l(y)$ is either a singleton or the empty set.

9. $T_{Y S}$ if for each $x, y \in X(x \neq y), C l(x) \cap C l(y)$ is either $\emptyset$ or $x$ or $y$.

From the notions defined above it is obtained the following diagram of strict implications ([1]).

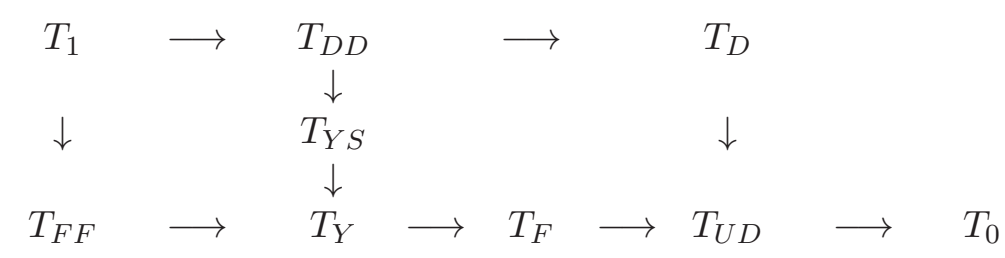

The above axioms can be characterized in terms of the derived, the closure, the shell and the kernel of singletons ([1]). The kernel of $A \subseteq X$, denoted by $\widehat{A}$, is defined as the intersection of the open sets containing $A$ and the shell of $A$, denoted by $\hat{A}$, as $\widehat{A} \backslash A$.

Definition 2. A minimal structure or an $m$-structure on the nonempty set $X$, is a class $m$ of subsets of $X$ such that $\emptyset, X \in m$.

For a nonempty set $X$ and an $m$-structure $m$ on $X$, the pair $(X, m)$ is called $m$-space. Each element of $m$ is said to be $m$-open set and the complement of an $m$-open set is an $m$-closed set. Note that the union and intersection of $m$-open sets are not generally $m$-open sets; thus each topological space is an $m$-space and it is easy to see that there exist $m$-spaces that are not topological spaces.

As in the topological case, if $X$ is an $m$-space and $A \subseteq X$ the $m$-closure of $A(m-C l(A))$ is the intersection of all $m$-closed sets containing $A$. It is clear that if $A$ is $m$-closed then $m-C l(A)=A$ but the converse is not true in general. We will say that the collection $m$ satisfies the Maki's condition ([4]) if $m$ is closed under unions. Thus under the Maki's condition the intersection of $m$-closed sets is an $m$-closed and hence also the $m$-closure of any subset. 
The $m$-derived set $(m-D e r)$ is defined similarly to the topological case and it is proved that $m-C l(A)=A \cup m-\operatorname{Der}(A)$ for any $A \subseteq X$.

We finish this section with some special classes of sets, which we will use to characterize some separation axioms in $m$-spaces.

Definition 3. Let $X$ be an $m$-space and $A \subseteq X$. Then:

1. The $m$-kernel of $A$ is $m-\widehat{A}=\bigcap\{G \subseteq X: G \in m, A \subseteq G\}$.

2. The $m$-shell of $A$ is $m-\widehat{A}=m-\widehat{A} \backslash A$.

3. $m-\langle A\rangle=m-C l(A) \cap m-\widehat{A}$.

The following properties of the notions defined above will be used in the final section.

Proposition 4. Let $X$ be an $m$-space and $x, y \in X$. The following statements hold:

1. If $y \in m-\widehat{x}$, then $m-\widehat{y} \subseteq m-\widehat{x}$.

2. $y \in m-\widehat{x}$ iff $x \in m-C l(y)$.

3. $y \in m-\hat{x}$ iff $x \in m-\operatorname{Der}(y)$.

4. For each $p \in X, m-\hat{p}$ is either the empty set or a singleton iff for each $x, y \in X(x \neq y), m-\operatorname{Der}(x) \cap m-\operatorname{Der}(y)=\emptyset$.

5. If $y \in m-\langle x\rangle$, then $m-\langle y\rangle=m-\langle x\rangle$.

6. For each $x, y \in X$, either $m-\langle x\rangle \cap m-\langle y\rangle=\emptyset$ or $m-\langle x\rangle=m-\langle y\rangle$.

\section{Some Low Separation Axioms in $m$-Spaces}

Inspired by [1] we define some classes of $m$-spaces, which are related to $m-T_{0}$ and $m-T_{1}$ spaces. We recall that the $m$-space $X$ is called $m-T_{0}$ if for each $x, y \in X,(x \neq y)$ either $x \vdash y$ or $y \vdash x$; and it is said to be $m-T_{1}$ if for each $x, y \in X,(x \neq y)$ one has that $x \vdash y$ and $y \vdash x$.

Definition 5. The $m$-space $X$ is called:

1. $m-T_{D}$ if for each $x \in X, m-\operatorname{Der}(x)$ is $m$-closed. 
2. $m-T_{U D}$ if for each $x \in X, m-\operatorname{Der}(x)$ is the union of disjoint $m$-closed sets.

3. $m-T_{D D}$ if it is $m-T_{D}$ and for each $x, y \in X(x \neq y), m-\operatorname{Der}(x) \cap m-$ $\operatorname{Der}(y)=\emptyset$.

4. $m-T_{F}$ if for any point $x$ and any finite subset $F$ of $X$ such that $x \notin F$, either $x \vdash F$ or $F \vdash x$.

5. $m-T_{F F}$ if for any two finite subsets $F_{1}$ and $F_{2}$ of $X$ with $F_{1} \cap F_{2}=\emptyset$, either $F_{1} \vdash F_{2}$ or $F_{2} \vdash F_{1}$.

6. $m-T_{Y}$ if for each $x, y \in X(x \neq y), m-C l(x) \cap m-C l(y)$ is either a singleton or the empty set.

7. $m-T_{Y S}$ if for each $x, y \in X(x \neq y), m-C l(x) \cap m-C l(y)$ is either $\emptyset$ or $x$ or $y$.

8. $m-T(\alpha)$ if for each $y \in X, x \in m-\operatorname{Der}(y)$ implies $m-\operatorname{Der}(x)=\emptyset$.

In the following we will determine the relationship between the classes of $m$-spaces defined above. Note first that the implications $m-T_{1} \rightarrow m-T_{D D}$, $m-T_{D} \rightarrow m-T_{U D}$ and $m-T_{D D} \rightarrow m-T_{Y S}$ are evident.

Proposition 6. The following affirmations hold:

1. Every $m-T_{U D}$ space is $m-T_{0}$.

2. Every $m-T_{F F}$ space is $m-T_{Y}$.

3. Every $m-T_{F}$ space is $m-T(\alpha)$.

4. Every $m-T(\alpha)$ space is $m-T_{0}$.

5. Every $m-T_{Y}$ space is $m-T(\alpha)$.

Proof. 1. For $x, y \in X(x \neq y)$ if $y \in m-\operatorname{Der}(x)$, then there exists an $m$-closed set $H$ such that $x \in H^{c}$ and $y \notin H^{c}$. Since the other case is evident we conclude that $X$ is $m-T_{0}$.

2. If there exist $x, y \in X(x \neq y)$ such that the set $m-C l(x) \cap m-C l(y)$ is neither $\emptyset$ nor a singleton, then there exist $p, q \in X(p \neq q)$ such that $p, q \in$ $m-C l(x) \cap m-C l(y)$. If $\{p, q\} \cap\{x, y\}=\emptyset$ then for $F_{1}=\{p, x\}$ and $F_{2}=\{q, y\}$ we have that $F_{1} \forall F_{2}$ and $F_{2} \forall F_{1}$, a contradiction. If $p=x$ and $q \neq y$ then for $F_{1}=\{x\}, F_{2}=\{q, y\}$ is again obtained a contradiction. Finally, since the 
other cases are impossible, we conclude that $m-C l(x) \cap m-C l(y)$ is either $\emptyset$ or a singleton, that is, $X$ is $m-T_{Y}$.

3. Let $y \in X$ and $x \in m-\operatorname{Der}(y)$. If $p \in m-\operatorname{Der}(x)$ and $p=y$, then $F=\{y\} \forall x$ and $x \not \forall F$ which is a contradiction. The case $p \in m-\operatorname{Der}(x)$ and $p \neq y$ also leads to a contradiction. Hence $m-\operatorname{Der}(x)=\emptyset$ and thus $X$ is $m-T(\alpha)$.

4. Let $x, y \in X$ with $x \neq y$. If $x \in m-\operatorname{Der}(y)$, then by assumption $y \notin m-\operatorname{Der}(x)$ and the result follows. The other case is analogous, so that $X$ is $m-T_{0}$.

5. Let $y \in X$ and $x \in m-\operatorname{Der}(y)$. If $p \in m-\operatorname{Der}(x)$ and $p=y$, then $\{x, y\} \subseteq m-C l(x) \cap m-C l(y)$ which is a contradiction. Analogously the case $p \neq y$ leads to a contradiction. Hence $m-\operatorname{Der}(x)=\emptyset$ and thus $X$ is $m-T(\alpha)$.

The topological version of the $m-T(\alpha)$ notion was introduced in [1] (Theorem 3.2). However, it is equivalent to the notion $T_{F}$, which is not true in $m$-spaces (Example $7(2)$ ).

In the topological case the implications $T_{1} \rightarrow T_{F F}, T_{Y} \rightarrow T_{F}, T_{Y} \rightarrow T_{U D}$, $T_{F} \rightarrow T_{U D}$ hold. However, these implications are not true in general in $m$-spaces, as we show in the following example.

Example 7. 1. Consider $\mathbb{R}$ and $m=\{\emptyset, \mathbb{R}\} \cup\{\mathbb{R}-\{x\}: x \in \mathbb{R}\}$. It is clear that this space is $m-T_{1}$. Let $F_{1}=\{0,1\}, F_{2}=\{2,3\}$. The $m$-open sets containing $F_{1}$ have the form $\mathbb{R}-\{z\}$ with $z \neq 0,1$. If $z \neq 2,3$ then $\mathbb{R}-\{z\} \cap F_{2}=F_{2}$ and so $F_{1} \forall F_{2}$. If either $z=2$ or 3 then $\mathbb{R}-\{z\} \cap F_{2}=F_{2}-\{z\}$ and $F_{1} \nvdash F_{2}$. Analogously $F_{2} \nvdash F_{1}$ and so this space is not $m-T_{F F}$. In conclusion $m-T_{1} \nrightarrow \rightarrow m-T_{F F}$.

2. Let $X=\{1,2,3\}$ and $m=\{\emptyset, X,\{1\},\{1,2\},\{2,3\},\{3\}\}$. Since for each $x \in X, m-\operatorname{Der}(x)=\emptyset$ then this space is $m-T_{Y}$. For $2 \in X$ and $F=\{1,3\}$ we have that $\{2\} \forall F$ and $F \forall\{2\}$, so this space is not $m-T_{F}$. This example shows that $m-T_{Y} \nrightarrow \rightarrow m-T_{F}$ and $m-T(\alpha) \nrightarrow \rightarrow m-T_{F}$.

3. Consider $\mathbb{R}$ and $m=\{\emptyset, \mathbb{R}\} \cup\{A \subseteq \mathbb{R}: 0 \notin A, A$ is finite $\}$. If $x \neq 0$ then $m-\operatorname{Der}(x)=\{0\}$ and if $x=0$ then $m-\operatorname{Der}(0)=\emptyset$, so this space is $m-T_{Y}$. Since the nonempty $m$-closed sets are infinite, the set $m-\operatorname{Der}(x)=\{0\}$ can not be expressed as a union of disjoint $m$-closed sets. Then this space is not $m-T_{U D}$ and thus $m-T_{Y} \nrightarrow \rightarrow m-T_{U D}$.

4. Let $X$ be an infinite set, $p \in X$ a fixed element and $m=\{\emptyset, X\} \cup\{A \subseteq$ $X: p \in A,|A| \geq 3$ and $A$ is finite $\}$. Let $x \in X$ and $F$ a finite subset of $X$ with $x \notin F$. If $x=p$ then $p \vdash F$. If $x \neq p$ and $p \in F$ then $F \vdash x$ and if $p \notin F$ then $x \vdash F$. Hence this space is $m-T_{F}$. Since the set $m-\operatorname{Der}(p)=X-\{p\}$ 
can not be expressed as a union of disjoint $m$-closed sets we conclude that $X$ is not $m-T_{U D}$. Thus, $m-T_{F} \nrightarrow \rightarrow m-T_{U D}$ and $m-T(\alpha) \not \rightarrow m-T_{U D}$.

In conclusion we obtain the following diagram of implications.

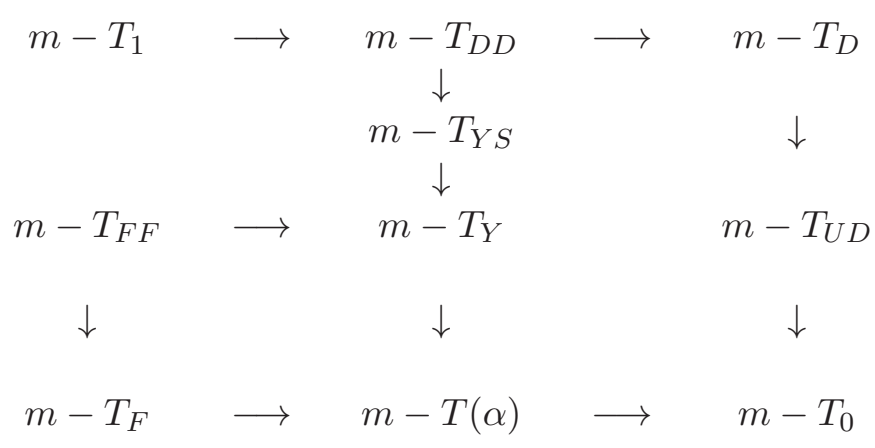

Since each topological space is in particular an $m$-space the implications $m-T_{1} \rightarrow m-T_{D D} \rightarrow m-T_{D} \rightarrow m-T_{U D} \rightarrow m-T_{0}, m-T_{D D} \rightarrow m-T_{Y S} \rightarrow$ $m-T_{Y}$ and $m-T_{F F} \rightarrow m-T_{Y}$ are strict. Moreover, since each $m-T_{Y}$ space is $m-T(\alpha)$ (Proposition $6(5)$ ) the Example 7 (2) shows that $m-T(\alpha) \nrightarrow \rightarrow m-T_{F}$. The remaining implications are also strict as we show below.

Example 8. 1. For $X=\{1,2,3,4\}$ and $m=\{\emptyset, X,\{1\},\{2\},\{1,2,3\},\{1,2$, $4\}\}$ we have that $m-\operatorname{Der}(1)=m-\operatorname{Der}(2)=\{3,4\}$ and $m-\operatorname{Der}(3)=$ $m-\operatorname{Der}(4)=\emptyset$. So, this space is $m-T(\alpha)$. Moreover, $m-C l(1) \cap m-C l(2)=$ $\{3,4\}$, that is, $X$ is not $m-T_{Y}$. Then $m-T(\alpha) \not \rightarrow m-T_{Y}$.

2. Consider $\mathbb{R}$ with $m=\{\emptyset, \mathbb{R}\} \cup\{[x, \infty): x \in \mathbb{R}\}$. It is clear that this space is $m-T_{0}$ and it is not $m-T(\alpha)$. Thus, $m-T_{0} \not \rightarrow m-T(\alpha)$.

3. Let $X$ be a set such that $|X| \geq 4$ and $m=\{\emptyset, X\} \cup\{\{x\}: x \in X\}$. For different elements $a, b, c, d \in X$ one has that $F_{1}=\{a, b\} \not F_{2}=\{c, d\}$ and $F_{2} \not$ $F_{1}$. So, this space is not $m-T_{F F}$ but it is $m-T_{F}$. Hence $m-T_{F} \not \rightarrow m-T_{F F}$.

Finally, it is easy to see that each topological space $T_{i}$ is an $m-T_{i}$ space and this implication is strict. Therefore, the class of topological spaces $T_{i}$ is strictly contained in the class of $m-T_{i}$ spaces.

\section{Characterizations}

In what follows, we use the concepts of $m$-derived, $m$-closure, $m$-shell and $m$-kernel to characterize some low separation axioms in $m$-spaces. We start with several characterizations of the $m-T_{0}$ spaces. This result extends the 
Theorem 2.3 of [1], the proof follows of the definitions and the application of Proposition 4.

Theorem 9. Let $X$ be an $m$-space. The following conditions are equivalent:

1. $X$ is $m-T_{0}$.

2. If $y \in m-C l(x)$ with $y \neq x$, then $x \notin m-C l(y)$.

3. If $y \in m-\operatorname{Der}(x)$, then $m-C l(y) \subseteq m-\operatorname{Der}(x)$.

4. If $y \in m-\widehat{x}$, then $m-\widehat{y} \subseteq m-\hat{x}$.

5. For each $x \in X, m-\operatorname{Der}(x) \cap m-\hat{x}=\emptyset$.

6. For each $x \in X, m-\langle x\rangle=x$.

In a similar way it can be obtained the following characterizations of the $m-T_{1}$ spaces. This result extends Theorem 2.4 of [1].

Theorem 10. Let $X$ be an $m$-space. The following conditions are equivalent:

1. $X$ is $m-T_{1}$.

2. For each $x \in X, m-C l(x)=x$.

3. For each $x \in X, m-\operatorname{Der}(x)=\emptyset$.

4. For each $x \in X, m-\widehat{x}=x$.

5. For each $x \in X, m-\hat{x}=\emptyset$.

6. For each $x, y \in X$ with $x \neq y, m-C l(x) \cap m-C l(y)=\emptyset$.

7. For each $x, y \in X$ with $x \neq y, m-\widehat{x} \cap m-\widehat{y}=\emptyset$.

8. $m-N_{D}=X$, where $m-N_{D}=\{x \in X: m-\operatorname{Der}(x)=\emptyset\}$.

9. $m-N_{S}=X$, where $m-N_{S}=\{x \in X: m-\hat{x}=\emptyset\}$.

It is well known that the topological spaces $T_{D}$ are characterized as those where each singleton is the intersection of a closed set with an open set ([1], Theorem 3.1). However, in the $m$-spaces this property is not true in general but if the Maki's condition is satisfied then the result is verified. 
Theorem 11. If $X$ is $m-T_{D}$, then each singleton is the intersection of an $m$-open set with the $m$-closure of some subset of $X$. The converse is true if $m$ satisfies the Maki's condition.

Proof. For each $x \in X$ we have that $(m-\operatorname{Der}(x))^{c} \cap(m-C l(x))=x$ and so the result follows.

Conversely, if for each $x \in X, x=G_{x} \cap m-C l\left(A_{x}\right)$ where $G_{x}$ is an $m$-open and $A_{x} \subseteq X$, then $x=G_{x} \cap m-C l(x)$ and so $m-\operatorname{Der}(x)=m-C l(x) \cap G_{x}^{c}$. Thus by the Maki's condition we obtain that $m-\operatorname{Der}(x)$ is $m$-closed.

In $m$-spaces the $m-T(\alpha)$ notion can be used to characterize the $m-T_{Y}$ axiom. This situation is analogous to the topological case where the $T_{F}$ notion is used ([1], Theorem 3.6).

Theorem 12. Let $X$ be an $m$-space. The following conditions are equivalent:

1. $X$ is $m-T_{Y}$.

2. $X$ is $m-T(\alpha)$ and for each $x, y \in X$ with $x \neq y$ the set $m-\operatorname{Der}(x) \cap$ $m-\operatorname{Der}(y)$ is either empty or a singleton.

3. $X$ is $m-T(\alpha)$ and for each $x, y \in X$ with $x \neq y$ the set $m-\hat{x} \cap m-\hat{y}$ is either empty or a singleton.

4. For each $x, y \in X$ with $x \neq y$ the set $m-\widehat{x} \cap m-\widehat{y}$ is either empty or a singleton.

Proof. The first implication is evident. For the remaining it is enough to reason by contradiction and to apply 3 and 4 of Proposition 4 .

Similar to the last theorem the $m-T(\alpha)$ notion can be used to characterize the $m-T_{Y S}$ axiom.

Theorem 13. $X$ is $m-T_{Y S}$ iff $X$ is $m-T(\alpha)$ and for each $x, y \in X$ with $x \neq y, m-\operatorname{Der}(x) \cap m-\operatorname{Der}(y)=\emptyset$.

Proof. It is clear that $X$ is $m-T(\alpha)$. If for each $x, y \in X(x \neq y)$ there exists $p \in m-\operatorname{Der}(x) \cap m-\operatorname{Der}(y)$, then $p \in m-C l(x) \cap m-C l(y)$, which is false. So the result follows.

Conversely, for each $x, y \in X(x \neq y)$ we have that $m-C l(x) \cap m-C l(y)=$ $(x \cap m-\operatorname{Der}(y)) \cup(y \cap m-\operatorname{Der}(x))$. Since $X$ es $m-T(\alpha)$, that union of sets is necessarily different from $\{x, y\}$. Thus $X$ is $m-T_{Y S}$. 
Theorem 14. Let $X$ be an $m$-space. If for each $x, y \in X(x \neq y)$, $m-\operatorname{Der}(x) \vdash m-\operatorname{Der}(y)$ or $m-\operatorname{Der}(y) \vdash m-\operatorname{Der}(x)$, then $X$ is $m-T_{Y S}$. The converse is true if for each $x \in X, m-C l(x)$ is $m$-closed.

Proof. If there exist $x, y \in X(x \neq y)$ and $p$ different from $x$ and $y$ such that $p \in m-C l(x) \cap m-C l(y)$, then $p \in m-\operatorname{Der}(x)$ and $p \in m-\operatorname{Der}(y)$. Thus we obtain a contradiction $m-\operatorname{Der}(x) \nvdash m-\operatorname{Der}(y)$ and $m-\operatorname{Der}(y) \nvdash m-\operatorname{Der}(x)$.

Conversely, if $m-C l(x) \cap m-C l(y)=\emptyset$ for $x \neq y$ then $m-\operatorname{Der}(y) \vdash$ $m-\operatorname{Der}(x)$ with the $m$-open set $(m-C l(x))^{c}$. If $m-C l(x) \cap m-C l(y)=x$, then $m-\operatorname{Der}(x) \vdash m-\operatorname{Der}(y)$ with the $m$-open set $(m-C l(y))^{c}$. Since the other case is analogous we obtain the result.

Note that the previous result suggests the existence of a new separation notion between $m-T_{D D}$ and $m-T_{Y S}$, since in each $m-T_{D D}$ space for each $x \neq y$ one has that $m-\operatorname{Der}(x) \vdash m-\operatorname{Der}(y)$ and $m-\operatorname{Der}(y) \vdash m-\operatorname{Der}(x)$.

We finish this work with the characterization of the $m-T(\alpha)$ notion, which extends Theorem 3.4 of [1] to $m$-spaces.

Theorem 15. Let $X$ be an $m$-space. The following conditions are equivalent:

1. $X$ is $m-T(\alpha)$.

2. For each $x, y \in X(x \neq y), m-\operatorname{Der}(x) \cap m-\hat{y}=\emptyset$.

3. $N_{S} \cup N_{D}=X$.

4. For each $x \in X, y \in m-\widehat{x}$ implies $m-\hat{y}=\emptyset$.

Proof. $1 \Rightarrow 2$. If $p \in m-\operatorname{Der}(x) \cap m-\hat{y}$ for $x \neq y$, then we would have $p \in m-\operatorname{Der}(x)$ and $y \in m-\operatorname{Der}(p)$ (Proposition 4 (4)), which contradicts 1. Thus 2 follows.

$2 \Rightarrow 3$. If there exists $x \in X$ such that $m-\operatorname{Der}(x) \neq \emptyset$ and $m-\hat{x} \neq \emptyset$, then there exist $p, q \in X(p \neq q)$ such that $x \in m-\widehat{p} \cap m-\operatorname{Der}(q)$ (Proposition 4 (4)), which contradicts 2 . Hence $N_{S} \cup N_{D}=X$.

$3 \Rightarrow 4$. If $y \in m-\widehat{x}$ and $m-\hat{y} \neq \emptyset$, then $x \in m-\operatorname{Der}(y)$ (Proposition 4 (4)) and by $3 m-\operatorname{Der}(y)=\emptyset$, which is a contradiction. Then 4 follows.

$4 \Rightarrow 1$. If $x \in m-\operatorname{Der}(y)$ and $m-\operatorname{Der}(x) \neq \emptyset$, then we would have $y \in m-\widehat{x}$ and $x \in m-\hat{p}$ for some $p \in X$ (Proposition 4 (4)), which contradicts 4. It follows that $X$ is $m-T(\alpha)$. 


\section{References}

[1] C.E. Aull, W. J. Thron, Separation axioms between $T_{0}$ and $T_{1}$, Indag. Math., 24 (1962), 26-37.

[2] C. Cao, B. Wang, W. Wang, Generalized topologies, generalized neighborhood systems, and generalized interior operators, Acta Math. Hungar., 132 (2011), 310-315.

[3] Á. Császár, Generalized open sets, Acta Math. Hungar., 75 (1997), 65-87.

[4] H. Maki, On generalizing semi-open and preopen sets, In: Report for Meeting on Topological Spaces Theory and its Applications, Yatsushiro College of Technology, 1996, 13-18.

[5] W.K. Min, Y.K. Kim, $M^{\star}$-continuity and product minimal structure on minimal structures, Int. J. Pure Appl. Math., 69 (2011), 329-339.

[6] A. Pushpalatha, E. Subha, Strongly generalized continuous functions in minimal structures, Int. J. Math. Sci. Eng. Appl., 4 (2010), 197-203.

[7] M.S. Sarsak, Weak separation axioms in generalized topological spaces, Acta Math. Hungar., 131 (2011), 110-121.

[8] P. Urysohn, Uber die matchtigkeit der zusammenhangenden mengen, Math. Ann., 94 (1925), 262-295.

[9] W.T. Van Est, H. Freudenthal, Trennung durch stetige funktionen in topologischen raumen, Ind. Math., 13 (1951), 359-368.

[10] X.E. Xun, X.E. Ying, $\mu$-Separations in generalized topological spaces, Appl. Math. J. Chinese Univ., 25 (2010), 243-252.

[11] W.T. Young, A note on separation actions and their application in the theory of a locally connected topological space, Bull. Amer. Math. Soc., 49 (1943), 383-385. 
